(c) Auteurs. Cette œuvre, disponible à http://dx.doi.org/10.18162/fp.2020.676, est distribuée sous licence Creative Commons Attribution 4.0 International http://creativecommons.org/licences/by/4.0/deed.fr

Gonzague Yerly

Université et Haute école pédagogique de Fribourg

(Suisse)

Dany Laveault

Université d'Ottawa (Canada)

\section{Évaluer les apprentissages en contexte de pandémie : aller au-delà de la notation pour soutenir la réussite de tous les élèves ${ }^{1}$}

Learning assessment in a pandemic context: beyond grading to foster academic success in all students

\section{ésumé}

La contribution de cet article au numéro thématique est de centrer l'analyse de la période de COVID-19 sur l'évaluation des apprentissages. La période écoulée s'est avérée en effet très particulière, car, dans la plupart des systèmes éducatifs, les notes certificatives ont été temporairement mises entre parenthèses. Ceci ne signifie pas pour autant que l'évaluation ne joue aucun rôle dans I'apprentissage des élèves, que ce soit avant, pendant ou après le confinement sanitaire. Au-delà du constat tiré de cette période très spéciale, la présente contribution propose des recommandations pour que l'évaluation des apprentissages joue pleinement son rôle dans ce nouveau contexte.

\section{Mots-clés}

COVID-19, évaluation des apprentissages, évaluation certificative, notes, évaluation-soutien d'apprentissage

Abstract

This article contributes to this thematic issue with an examination of learning assessment during the COVID-

19 crisis. The summer of 2020 was highly unusual in that most education systems put their certification assessments on hold. This does not mean that assessment had no part to play in student learning, either before, during, or after the confinement period. Beyond the observations made during this extraordinary period, recommendations are proposed to enable learning assessment to play its full role in this new context.

Keywords

COVID-19, learning assessment, certification assessment, grades, assessment for learning

\section{La période de COVID-19: une parenthèse sans notes dans les systèmes scolaires}

\section{Une période scolaire sans notes. Une situation unique.}

En mars 2020, la pandémie de COVID-19 a contraint la plupart des systèmes scolaires à fermer les portes des établissements, du primaire à l'université. Cette période aura été marquée par de nombreux changements et adaptations d'urgence touchant la formation des élèves et les pratiques professionnelles des enseignants ${ }^{2}$ à travers le monde. En matière d'évaluation des apprentissages, le semestre du printemps 2020 aura été marqué par un changement des « règles du jeu » en remettant à plus tard la notation et la certification des apprentissages, principalement pour une question d'« égalité des chances ».

L'utilité de l'évaluation certificative à l'aide d'un score (Mottier Lopez et Allal, 2008 ; Scallon, 2004) a souvent été considérée comme contreproductive par les chercheurs en éducation (Butera, 2011 ; Merle, 2015), car elle crée une culture du testing plutôt qu'une culture de l'évaluation et de l'apprentissage. Dans bien des juridictions scolaires, la période de mars à juin 2020 aura représenté une formidable occasion pour les élèves, les enseignants et les systèmes éducatifs d'expérimenter l'apprentissage et l'enseignement sans la contrainte des notes à des fins de certification. Cette expérience s'est d'abord faite dans les conditions peu familières de l'enseignement à distance. Puis, à la levée du confinement, plusieurs écoles ont rouvert leurs portes, mais sans notes. 
Le vécu des enseignants durant cette parenthèse scolaire sans notes et son impact sur les systèmes scolaires ont permis de nouvelles prises de conscience au sujet des pratiques d'évaluation des apprentissages et suscité un nouveau questionnement sur le rôle que peut jouer l'évaluation dans le nouveau contexte créé par la pandémie.

Ce texte présente tout d'abord une réflexion théorique, un bref rappel des principaux travaux traitant de la question de la note et de ses effets sur l'apprentissage et l'enseignement. Nous étendons également notre réflexion au rôle des pratiques évaluatives des enseignants dans le processus d'apprentissage des élèves et proposons une série de recommandations pour les élèves, les enseignants et les systèmes scolaires concernant le rôle que celles-ci peuvent jouer pour appuyer l'enseignement et soutenir l'apprentissage des élèves.

\section{L'abandon momentané des notes comme solution aux inégalités renforcées par la période de COVID-19}

Dans de nombreux systèmes scolaires, la pandémie de COVID-19 aura poussé les autorités à supprimer momentanément le recours à la note "certificative». En effet, nous différencions conceptuellement évaluation «certificative » et «sommative » (Mottier Lopez et Allal,2008; Roegiers, 2004; Scallon, 2004), la note nétant pas forcément un indicateur « qui compte » pour la certification de l'élève. Lévaluation « certificative » sert à faire un bilan, à porter un jugement global sur les acquis des élèves après une période de formation afin de prendre une décision administrative telle que la diplomation des élèves (attribuer ou non un " certificat»), leur promotion ou non dans la suite du cursus, etc. Lévaluation certificative suit donc principalement une fonction administrative, elle fait le lien entre la classe et le système scolaire. Elle ne suit aucune finalité de régulation de l'apprentissage et de l'enseignement. L'évaluation "sommative " sert également à dresser un bilan des acquis des élèves et est interne à la classe et à la relation pédagogique. Elle est avant tout un type de recueil de l'information qui permet à l'enseignant, par différents moyens, de porter « un tableau d'ensemble » (Scallon, 2004,p. 206) des acquis de l'élève à des moments importants de sa formation. Les données d'apprentissage recueillies peuvent donc servir d'un côté à la régulation de l'apprentissage et de l'enseignement (actions pédagogiques et didactiques) et, d'un autre côté, ces données peuvent contribuer à l'évaluation certificative.

La décision de reporter momentanément le recours aux notes certificatives durant la pandémie a été prise pour des raisons d' "égalité des chances ", voire d'« égalité de traitement " entre les élèves (Crahay, 2012). La situation sanitaire a en effet accru les disparités entre les élèves. Au-delà des différences présentes habituellement, de nouvelles disparités se sont ajoutées : différences de réactions émotionnelles envers la crise sanitaire, fracture dans l'accès au matériel informatique, hétérogénéité des compétences numériques, mais aussi de gestion du temps et des ressources, situations très inégales quant aux possibilités de soutien en milieu familial, etc.

Même sans notes, une décision devait être prise quant à la promotion des élèves à l'année suivante, dans le cycle suivant ou pour les élèves au terme de leur formation, pour l'accession au diplôme. Selon les juridictions scolaires et les conditions qui y prévalaient, cette prise de décision visant la certification a pris plusieurs formes où les notes sont intervenues de manière plus ou moins décisive :

2 - Formation et profession 28(4 hors-série), 2020 
- Maintien des procédures d'évaluation certificative et des notes, avec des modalités de circonstance (à distance ou non). Lorsqu'aucun indicateur ne pouvait être pris en compte pour la diplomation (par ex. : pas de notes ou de traces d'apprentissages en cours d'année), certains examens ont été maintenus, organisés à distance ou en présence avec des mesures sanitaires adéquates.

- Maintien des procédures d'évaluation certificative et des notes, avec des aménagements liés aux conséquences des résultats. Dans certaines universités suisses, les examens ont bien eu lieu selon des modalités adaptées, mais sans possibilités d'échec.

- Maintien des procédures d'évaluation certificative, sans note. Les examens ou autres procédures d'évaluation étaient adaptés à la situation et les résultats ont été exprimés sous une autre forme qu'un résultat chiffré (par ex. : acquis vs non acquis, validé vs non validé) et accompagnés d'un feedback (ou non).

- Recours aux notes les plus récentes et à d'autres indicateurs. Dans certains cas, les notes déjà existantes avant la pandémie ont été prises en compte pour la promotion et/ou diplomation des élèves. Certaines juridictions ont procédé à des "modérations statistiques » (Laveault \& Yerly, 2017), les résultats des années précédentes étant pris en compte notamment en Australie, NouvelleZélande, Pays de Galles.

- Abandon des procédures d'évaluation certificative et recours accru au jugement professionnel des enseignants. Certaines juridictions ont préféré s'en remettre essentiellement au «jugement professionnel » des enseignants sans recours à des examens faits en classe ou à des épreuves standardisées (par ex. : en Écosse, aux É.-U.).

- Absence totale de toute forme d'évaluation certificative, certification automatique. En Suisse romande - tout comme au Québec -, tous les systèmes cantonaux de scolarité obligatoire ont supprimé les notes, les bulletins indiquant que l'année a été suivie. Tous les élèves sont promus à l'année suivante. Les épreuves externes cantonales ont été annulées ou proposées comme exercice. En outre, à l'heure actuelle, certains cantons ont déjà annoncé que le début de l'année scolaire (automne 2020) serait sans note dans un premier temps (par ex. : les 6 premières semaines).

Malgré les différentes configurations exposées ci-dessus, la pandémie de COVID-19 a largement modifié les règles en matière d'évaluation certificative pour les élèves, les enseignants et les systèmes scolaires. Pour bien des juridictions scolaires à travers le monde, l'arrêt momentané des notes certificatives aura permis aux différents acteurs de vivre une période de confinement et d'enseignement à distance, puis un retour à l'école sans la pression de l'évaluation certificative. Comment a été vécue cette période inédite? Quel en a été l'impact sur la motivation, le rythme d'apprentissage et la participation des élèves? Quelles pratiques d'évaluation les enseignants ont-ils mises en œuvre dans cette période? Quel sera l'impact de cette période sans notes sur les systèmes scolaires et surtout, sur l'apprentissage des élèves? Quelles pratiques d'évaluation prioriser pour la suite et un retour progressif vers une situation plus « normale »? 


\section{Apprendre, enseigner et évaluer sans la pression des notes}

\section{Les effets de la note sur l'apprentissage et l'enseignement}

La notation chiffrée est devenue et reste un des piliers du fonctionnement actuel de l'École. Pourtant, ses conséquences sont décriées scientifiquement depuis des décennies (Merle, 2015). Malgré ces constats, la note reste majoritairement défendue dans la société civile, même par les enseignants ${ }^{2}$. Pour Butera (2011) et Merle (2014), il existe plusieurs idées reçues sur les notes. Ces auteurs illustrent leur manque de fondement. Nous complétons leur analyse avec d'autres travaux :

- La note est une mesure simple et claire de l'apprentissage. Certes, la note est un outil familier, facile de compréhension et largement répandu (Hadji, 2017). Toutefois, la note mesure une performance à un instant précis. Elle ne tient pas compte du processus d'apprentissage de l'élève et est marquée de nombreux biais (Butera, 2011). Pour Hadji (2017), la note - et plus encore la note chiffrée - crée l'illusion d'une mesure de l'apprentissage, au même titre que des échelles à intervalles égaux comme les échelles de température ou de quantités physiques. Nous ajoutons que l'usage de notes au dixième ou d'échelles de mesure en pourcentage renforce encore cette illusion de précision. Enfin, la note ne permet pas de fournir un feedback détaillé et précis puisqu'elle amalgame une variété de résultats d'apprentissage fort disparates. Elle n'indique pas clairement ce qu'il faut améliorer ni les objectifs à poursuivre par l'élève. Elle demeure surtout un outil qui sert à sanctionner, certifier et sélectionner de manière rapide, et peu à apprendre et à enseigner. Elle reste surtout liée à une conception «normative" de l'évaluation (Butera, 2011) qui sert davantage à situer la performance de chaque élève par rapport à celle des autres élèves de son groupe plutôt que par rapport à sa propre progression en rapport avec les objectifs du curriculum. Elle contribue au maintien d'une culture du testing (Birenbaum, 2016) au détriment d'une culture de l'apprentissage.

- La note est un instrument de motivation extrinsèque pour l'élève. La note peut être considérée comme un renforçateur positif lorsqu'elle est bonne ou comme un renforçateur négatif lorsqu'elle est mauvaise. Elle est souvent utilisée par les enseignants comme une récompense ou une punition (Butera, 2011). Merle (2014) ajoute que selon certains enseignants, les élèves travailleraient moins, voire pas du tout sans la note. Il dément l'idée préconçue que la plupart des élèves ne s'engageraient pas dans leurs activités scolaires sans note. Il donne pour exemple le système éducatif finlandais dans lequel les notes sont peu présentes dans les classes, mais où les résultats aux épreuves internationales à grande échelle sont parmi les meilleurs. Au bout du compte, les seuls à être motivés par la note seraient les élèves dont les scores sont déjà très élevés.

- La note reproduit une certaine compétition que les élèves expérimenteront dans la suite de leur parcours professionnel. Butera (2011) montre que la compétition liée aux notes développe des comportements antisociaux (par ex. : tricherie) et contribue à l'échec scolaire. Les mauvaises notes donnent une image négative de soi, développent un sentiment d'incompétence et influencent négativement les processus d'apprentissage (Merle, 2014). Elle pousse l'élève à travailler " pour la note », en poursuivant des buts de performance de préférence à des buts d'apprentissage (Viau, 2009). Elle pousse l'enseignant au «teaching to the test » (Yerly, 2017).

4 - Formation et profession 28(4 hors-série), 2020 
- La note représente une récompense juste au mérite. Cette affirmation ne tient pas, car généralement le résultat chiffré ne tient pas compte de l'état initial et de la progression des élèves, mais bien d'une performance finale. Ainsi, on ne considère pas la disparité de départ, telle que les préacquis des élèves, leur origine sociale, leurs accès aux ressources, etc. (Butera, 2011). En outre, ce mécanisme méritocratique les pousse à s'attribuer une « valeur scolaire » et les stigmatise (Hadji, 2017). Elle contribue ainsi grandement au décrochage et à l'échec scolaire (Merle, 2014).

Malgré toutes ces pièces à charge, nous pensons, à l'instar de Hadji (2017), que la note n'est pas toujours à la base du problème, mais qu'elle y contribue fortement. La note, combinée à d'autres indicateurs, peut rendre possible l'expression d'un « jugement d'acceptabilité » ainsi qu'une « lecture analytique » de la progression des élèves, sans prétendre à une mesure parfaitement objective d'un niveau de maîtrise : «Évaluer ne signifie pas s'épuiser à poursuivre la chimère de la note “vraie” ( Hadji, 2017, p. 244).

\section{Le rôle des pratiques évaluatives des enseignants dans le processus d'apprentissage des élèves}

Les pratiques évaluatives des enseignants dépendent à la fois de la culture évaluative collective (lois, règlements, formation, prescriptions implicites des juridictions scolaires et des établissements scolaires), mais aussi de leur conception personnelle quant à l'enseignement et de l'apprentissage.

De nombreux travaux de recherche ont démontré que des pratiques élaborées d'évaluation formative, c'est-à-dire une évaluation-soutien d'apprentissage (assessment for learning) (Laveault et Allal, 2016), sont parmi les plus puissants facteurs favorisant l'apprentissage sur le long terme et de manière autonome (Hattie, 2012). Par ses actions évaluatives, l'enseignant peut favoriser l'apprentissage des élèves en situant leurs acquisitions par rapport aux critères préétablis, en faisant ressortir les réussites et les difficultés des élèves, en fournissant des rétroactions détaillées et en mettant en œuvre une action différenciée auprès des élèves. L'évaluation permet également à l'enseignant d'ajuster sa planification et son enseignement. Cependant cette régulation ne peut être effective que si elle se fait de concert avec l'élève. L'apprenant doit mettre en œuvre et développer ses capacités d'autorégulation (Laveault, 2007; Cartier et Butler, 2016; Clark, 2012) parmi lesquelles l'autoévaluation est une compétence primordiale qui ne peut s'acquérir qu'en impliquant graduellement l'élève dans le processus (assessment as learning) (Earl, 2012). Lévaluation, tant formative que sommative, doit permettre à l'élève, soutenu par l'enseignant, d'apprendre à se fixer des buts d'apprentissage réalistes, à comprendre les critères de réussite, à monitorer lui-même sa progression et à agir sur son apprentissage (par ex. : changer de stratégie, ajuster ses objectifs, etc.). L'évaluation joue alors un rôle constructif à la fois sur la cognition, mais aussi sur la motivation des apprenants.

\section{Que retenir du premier choc causé par la pandémie par rapport aux pratiques d'évaluation? Quels sont les défis à relever pour la suite?}

\section{Une expérience sans notes de courte durée, le retour de la certification}

La période de pandémie de COVID-19 aura permis aux enseignants d'éprouver comment il est possible d'enseigner et d'évaluer les apprentissages des élèves sans le recours à la notation chiffrée (à distance, puis en présence pour certains). Nos différentes observations et expériences, notamment étayées par 
un sondage auprès d'étudiants-stagiaires suisses, montrent qu'une grande part des enseignants étaient soulagés de ne pas devoir mener d'évaluation certificative (ou différemment dans certains cas), d'une part parce que l'enseignement s'est fait dans des situations inhabituelles (à distance) et d'autre part parce que les résultats allaient être inégaux eu égard à la situation de confinement. Nos différentes observations montrent que, lors de la période d'enseignement à distance, la collecte d'information approfondie sur la progression des élèves était difficile pour les enseignants et peu effective. À la reprise de l'école en présentiel, les écarts creusés par la période d'enseignement à distance ont demandé beaucoup d'observations et de différenciations de la part des enseignants. Lors de cette période de reprise, le temps habituellement consacré à l'évaluation à visée de certification (répétitions, passation de tests, corrections, etc.) a pu être consacré plutôt à sa visée de régulation. Enfin, si certains enseignants - surtout dans les plus hauts degrés de la scolarité - ont constaté une baisse de la motivation des élèves sans notes certificatives et se sont sentis démunis sans la "carotte et le bâton ", d'autres ont pu profiter de l'opportunité d'enseigner dans un climat plus favorable à l'apprentissage. En effet, ce fut l'occasion pour eux de développer des pratiques d'enseignement plus ludiques et collaboratives, stimulant davantage la motivation intrinsèque des élèves.

Cette période sans notes certificatives aura été de courte durée. Lors de l'année scolaire qui suivra (avec ou sans nouvelle vague de la pandémie), il faudra à nouveau évaluer de manière certificative et la pression de ne pas prendre de retard dans le curriculum sera plus grande. En effet, une grande partie du confinement s'est caractérisée par le recours à des activités visant davantage à consolider les apprentissages. Une telle mesure est acceptable lorsqu'il faut temporiser ou gagner du temps. Elle n'est

plus envisageable si les mesures sanitaires doivent perdurer pendant un an ou plus et si l'on souhaite que les écarts entre élèves ne s'agrandissent pas davantage.

\section{Développer le potentiel de chacun, malgré les écarts entre élèves}

Le principal défi du retour à l'école sera d'aplanir et de réduire les écarts entre élèves en s'assurant de la maîtrise des prérequis essentiels à la poursuite du curriculum officiel, tout en développant le potentiel de chacun. Il faudra également garantir, par la suite, l'atteinte des attentes officielles (avec ou sans nouveau confinement, avec des mesures sanitaires spéciales, etc.) et la qualité du système, notamment des diplômes décernés. À la reprise, les élèves seront donc plus ou moins en rattrapage, et ce, différemment, selon que les apprentissages et la formation reçue durant la période de confinement auront permis de maintenir et d'acquérir les prérequis essentiels. Les programmes d'études sont habituellement fort chargés, mais ils sont aussi fort heureusement construits sous forme d'une structure spiralaire dans laquelle les objectifs d'apprentissage et/ou compétences sont atteints sur une période pouvant s'étendre sur plusieurs années. On peut donc tenter de mieux situer chaque élève par rapport aux objectifs du programme et se concentrer sur ce qu'il est prioritaire de mettre à jour.

C'est surtout à la rentrée scolaire que l'absence d'évaluation certificative et le manque d'évaluation formative au cours de la période écoulée d'enseignement à distance risquent de se faire sentir le plus lourdement. Sur quel bilan d'apprentissage pourra-t-on fonder les décisions administratives et pédagogiques quant à la constitution des groupes-classes et/ou des grappes à l'intérieur de chaque classe (si nécessaire par mesure sanitaire) ? Comment apporter une aide différenciée aux élèves selon leur profil d'apprentissage, mais aussi quel est ce profil, comment le déterminer?

6 - Formation et profession 28(4 hors-série), 2020 


\section{Quel rôle pour l'évaluation des apprentissages lors d'un retour progressif à la « normalité »?}

\section{L'importance de l'évaluation-soutien d'apprentissage pour redémarrer et repenser l'enseignement et l'apprentissage}

La crise pandémique aura démontré l'importance de fonder les décisions sanitaires sur des tests fréquents quant à la présence du coronavirus dans la population. Les pays qui se sont le mieux tirés d'affaire sont ceux qui avaient mis en place des mesures de dépistage rapides, efficaces et étendues. Au contraire, les pays qui ont tardé à prendre de telles mesures ont peiné à contrôler la propagation du virus. En matière d'éducation, la situation n'est pas différente. L'évaluation des apprentissages représente un moyen d'agir sur les écarts entre les élèves pendant que la remédiation demeure possible.

L'évaluation est appelée à jouer plus que jamais son rôle de soutien à l'apprentissage et à l'enseignement afin de développer le potentiel de tous les élèves, et en particulier envers ceux en difficulté d'apprentissage. Ceci semble signifier pour l'instant de continuer à temporiser sur un certain nombre de pratiques habituelles, mais aussi d'innover et d'adapter les pratiques aux circonstances nouvelles qui risquent de se présenter. Nous savons déjà que l'évaluation-soutien d'apprentissage (Laveault et Allal,2016) est, parmi les variables à la disposition des enseignants, celle qui a le plus grand potentiel de faire progresser les élèves (Hattie, 2012). C'est pourquoi elle mérite de figurer parmi les pratiques enseignantes susceptibles de soutenir l'apprentissage des élèves en présentiel, mais aussi à distance. Pour que l'évaluation-soutien d'apprentissage puisse jouer pleinement son rôle, il faut cependant relever de nouveaux défis, rencontrer un certain nombre de conditions et revoir certaines pratiques en matière d'évaluation.

- Revoir l'équilibre délicat entre les finalités de l'évaluation. L'expérience vécue au printemps 2020 avec la suspension temporaire de l'évaluation certificative a permis de prendre conscience à quel point les systèmes de notation pour assurer la certification, mais aussi pour motiver les élèves à performer, pouvaient avoir des effets non désirables. Cette dépendance à la note peut avoir un effet toxique et nuire à l'apprentissage, surtout dans des situations de formation à distance (en ligne ou autrement) où les inégalités sont renforcées et où il faut compter sur une plus grande autonomie de l'élève et sur une plus grande capacité à s'automotiver. Lévaluation certificative n'est pas nuisible ni condamnable en soi. C'est plutôt au trop grand déséquilibre entre les deux rôles de l'évaluation qu'il faut s'attaquer. L'évaluation à visée de certification pourrait être suspendue encore pour un temps, jusqu'à ce que tous les élèves aient eu l'opportunité de se mettre minimalement à niveau dans leur progression individuelle des apprentissages prévus au programme. Cette mesure est non seulement plus équitable, mais rendra aussi plus facile l'enseignement à des groupes d'élèves si les écarts entre eux ont été amoindris. La période est donc davantage propice à « se rendre compte plutôt qu'à rendre des comptes » (Yerly et Issaieva, in press)! Toutefois, si l'évaluation à visée de certification doit être mise sur pause, ceci ne signifie pas que l'évaluation n'a pas de rôle important à jouer.

- Une évaluation sommative (évaluation-bilan) au service des apprentissages. Nous sommes habitués à ce que l'évaluation sommative soit terminale, à visée de certification et notée. L'évaluation sommative n'a pas à être cela. Dans le contexte du retour en classe après une période plus ou moins longue de confinement, une évaluation sommative qui permet un bilan nuancé 
des apprentissages et particulièrement des prérequis s'avère d'autant plus nécessaire pour assurer le retour à une situation où l'enseignement à des groupes d'élèves pourra porter ses fruits. Une évaluation sommative à l'origine de tels bilans d'apprentissage a le potentiel d'apporter une forme de soutien à l'apprentissage des élèves. Pour y parvenir, il faudrait que le rôle de l'évaluation sommative, ou " évaluation-bilan ", puisse être élargi de la façon suivante. Elle pourrait avoir lieu au tout début de l'année scolaire afin d'identifier les priorités et exercer une influence sur la planification de l'enseignement. Cette évaluation-bilan n'a pas à être notée. Un score global pourrait être remplacé par un profil permettant de mieux différencier les forces et les faiblesses dans l'apprentissage des élèves et de mieux cibler les objectifs d'apprentissage à atteindre en priorité. Des activités d'évaluation-bilan peuvent avoir lieu à la maison, avec ou sans supervision des parents. Elles peuvent être aussi corrigées par les parents et les élèves eux-mêmes en vue d'établir leur propre profil et leurs propres cibles d'apprentissage. Somme toute et dit autrement, l'évaluation sommative, lorsqu'elle permet de réaliser un bilan détaillé des apprentissages (avec ou sans note d'ailleurs), peut jouer un rôle de soutien à l'apprentissage. Cependant, débuter l'année par une révision en bloc de la matière serait contre-productif (RECIT,2020). D'après les résultats d'une étude menée à la Nouvelle-Orléans après la dévastation occasionnée par l'ouragan Katrina, les initiatives les plus profitables semblent être celles où « les contenus prérequis (ceux qui devaient être vus l'année précédente) étaient abordés au fur et à mesure qu'ils étaient nécessaires durant l'année ». On voit que de cette manière, l'évaluation des prérequis permet de mieux atteindre les objectifs d'apprentissage lorsque leur rappel se fait au moment où ils doivent être mobilisés.

- Développer une culture de l'apprentissage en impliquantl'élève dansle processus d'évaluation. Une culture du testing axée uniquement sur la performance et la note favorise des résultats obtenus rapidement et des apprentissages superficiels soutenus par des pratiques de "teaching to the test ». Une telle culture ne contribue pas à réduire les écarts entre les élèves, au contraire. Elle favorise les élèves les plus performants et les plus compétitifs. Dans un tel environnement, l'évaluation entre pairs qui consiste à faciliter les échanges de rétroactions entre élèves, l'apprentissage collaboratif et le travail en équipe ne parviennent pas à s'épanouir. Développer une culture de l'apprentissage passe par l'implication de l'élève dans le processus évaluatif. Évaluer est une compétence qui s'acquiert, qui permet à l'élève de surveiller de plus près son activité d'apprentissage, de se fixer des cibles de réussite à atteindre et de modifier son activité, avec ou sans aide de l'enseignant ou de ses pairs, pour atteindre ses objectifs. En ce sens, elle fait partie des compétences métacognitives qui permettent à l'élève non seulement d'être plus autonome, mais aussi de collaborer activement avec l'enseignant. Plus l'élève est conscient de ses difficultés, plus il parvient à les formuler pour faciliter la recherche d'une solution aux difficultés auxquelles il fait face. Toutefois, les élèves habitués, par une culture du testing, à faire des efforts lorsque « ça compte » et pour qui la source de motivation est principalement extrinsèque peuvent ne pas s'investir sérieusement dans une telle démarche. Selon que la note a été plus ou moins utilisée dans le passé pour motiver les élèves à s'investir dans leur apprentissage, l'établissement d'une évaluation à des fins de régulation des apprentissages se fera plus ou moins facilement. Pourtant, nous savons - et les jeux vidéo nous le démontrent chaque jour - que les enfants aiment relever des défis à leur mesure et se fixer des cibles qu'ils sont en mesure d'atteindre. Il incombe donc aux enseignants de trouver des stratégies pour faire entrer progressivement les élèves dans ce processus, en leur offrant des opportunités d'autoévaluation par exemple.

- Une évaluation d'autant plus alignée sur les programmes d'études. À la reprise, l'évaluation des 
apprentissages menée par les enseignants devra plus que jamais être critériée et non normative (ce qui devrait d'ailleurs toujours être le cas). Lévaluation devra être très proche des attentes des curricula et non pas se baser sur une comparaison entre les élèves. D'abord, parce qu'il est inéquitable et inutile de comparer les résultats d'un élève aux résultats des autres élèves, et ce, d'autant plus lorsque les conditions de réussite sont inégales. Deuxièmement, parce que de telles formes d'évaluation ne fournissent aucune information sur ce qu'il faut faire, notamment sur les résultats d'apprentissage acquis et/ou à acquérir en priorité. Enfin, plus que tout autre facteur, la maîtrise des prérequis d'apprentissage est le meilleur prédicteur de la réussite scolaire (Viau, 2009). Pour s'assurer du meilleur « alignement » possible (Anderson, 2002), l'enseignant dispose de sa connaissance du programme de formation pour les élèves du niveau auquel il enseigne, mais aussi des acquisitions qui doivent avoir déjà été faites, celles qui demandent à être consolidées et celles qui doivent préparer à réussir l'année suivante. Les enseignants titulaires de niveaux conjoints peuvent déjà s'assurer du meilleur alignement possible en comparant leurs interprétations des programmes d'études et leurs planifications des apprentissages des élèves. En période de pandémie, le besoin d'alignement est d'autant plus grand que la pression exercée par les tests standardisés n'existe plus (Yerly, 2017). Heureusement, les programmes d'études prévoient une certaine reprise spiralaire des objectifs d'apprentissage. Afin d'assurer un certain « alignement ", les enseignants pourront pratiquer l'évaluation à rebours, qui consiste à planifier les activités d'enseignement et apprentissage à partir des résultats attendus à la fin de la formation et de structurer la progression des activités et des leçons en fonction de ces résultats.

- Se servir de l'évaluation comme d'un outil de communication avec les élèves et les parents. Lorsque les élèves reçoivent leur bulletin scolaire, il est déjà trop tard pour corriger quoi que ce soit. Les résultats de recherche ont amplement démontré que les élèves qui performent le mieux sont ceux qui possèdent la représentation la plus claire et la plus précise de ce que l'on attend d'eux (Laveault et Allal, 2016). Les meilleurs élèves parviennent même à anticiper les questions qui feront l'objet des examens. La communication des attentes et des résultats doit se faire de façon continue à toutes les étapes importantes de l'apprentissage de façon à ce que tous les élèves développent une représentation commune de ce qui est attendu. Difficile d'imaginer comment l'élève pourrait faire preuve de plus d'autonomie et gérer les processus cognitifs en jeu dans son apprentissage s'il ne possède pas une représentation claire du sens de l'apprentissage à réaliser et du résultat attendu. Au niveau primaire, voire au secondaire, la communication des attentes concerne aussi les parents si l'on souhaite qu'ils puissent jouer leur rôle dans le soutien de l'élève, d'autant plus lors d'une période d'enseignement à domicile. Plusieurs moyens sont à la disposition des enseignants pour rendre plus concrètes les cibles d'apprentissage :

- Analyser les critères à atteindre selon les plans d'études (seul et en équipe) ;

- Faire participer les élèves à l'élaboration des critères d'évaluation dès le début de l'apprentissage ;

- Spécifier les attentes et donner des exemples de ce à quoi serviront les nouveaux apprentissages ;

- Illustrer différents niveaux de réussite au moyen d'exemples prototypiques (par ex. : des « copies types ») de travaux d'élèves ;

- Utiliser les critères pour progresser par l'autoévaluation, évaluation entre pairs, etc.;

- Donner des feedback précis grâce aux critères. 


\section{Et plus concrètement? Comment mettre en place une évaluation qui soutient les apprentissages?}

Les voies d'exploration pour mieux redémarrer l'évaluation scolaire et l'adapter à la situation actuelle créée par la pandémie ne nous sont pas totalement inconnues de par nos expériences passées ou récentes. Pour parvenir à implanter avec succès une évaluation qui soutienne l'apprentissage, il faudra développer de nouvelles synergies et porter attention à plusieurs facteurs.

- Fournir du soutien... aux enseignants également. Si l'on veut que l'évaluation soutienne les apprentissages, il faut commencer par soutenir les enseignants. À juste titre, les enseignants peuvent être préoccupés par la charge de travail que représentera, lors de la prochaine rentrée scolaire, le redémarrage de l'enseignement avec des mesures sanitaires spéciales et la crainte d'une nouvelle vague de virus. En ce qui concerne l'évaluation, le bilan des apprentissages de tous les élèves et la régulation à mettre en place durant leur remise à niveau vont prendre un temps conséquent. Tant que dureront les mesures sanitaires, il est à prévoir que la charge globale de travail des enseignants devra être réaménagée afin de ne pas alourdir inutilement leur tâche. Si, comme nous le proposons, la certification était encore reportée un peu, le temps libéré pourrait être utilisé à bon escient, notamment pour favoriser la concertation entre enseignants en matière d'objectifs d'apprentissage, de critères de réussite et d'évaluation des apprentissages des élèves, de planification, etc. Il serait nécessaire que les autorités les soutiennent en facilitant le travail de concertation et en leur proposant des outils d'évaluation arrimés aux plans d'études (par ex. : d'anciennes épreuves externes à large échelle). Les enseignants pourraient avoir à disposition des épreuves diagnostiques préparées par les experts des programmes pour établir de tels bilans. C'est ce qui se produit en Nouvelle-Zélande où il n'existe pas de tests standardisés ni de publication des résultats des écoles. Les enseignants peuvent cependant avoir recours à des épreuves normées au moment de leur choix pour évaluer si le rendement de leurs élèves est conforme aux attentes du programme d'études officiel en matière de contenus et de niveaux d'exigence. Les enseignants n'ont pas à concevoir ces situations d'apprentissage, ni à les corriger, ni à les noter. Le tout se fait en ligne. Dans un tel contexte, le rôle principal de l'enseignant consiste à interpréter ces bilans et à offrir à chaque élève une rétroaction et des activités d'apprentissage complémentaires qui lui permettront de combler les lacunes dans sa formation. En outre, de telles évaluations-bilans nont pas forcément à être réalisées sur le temps de classe. Elles peuvent également se faire à la maison sous la supervision des parents pour les plus jeunes ou de façon autonome pour les élèves du secondaire.

- Le développement professionnel des enseignants et l'apprentissage collaboratif. Plus que jamais, étant donné le caractère nouveau de la situation des écoles dans le contexte de la crise sanitaire, le développement professionnel des enseignants et le travail collaboratif n'auront été aussi importants. Les écoles qui ont déjà constitué des communautés d'apprentissage professionnel (CAP) pourront mobiliser plus rapidement, que ce soit en ligne ou à l'école, le travail en collaboration et identifier les besoins de formation continue. Le travail en collaboration, les CAP et le développement professionnel peuvent et surtout doivent se poursuivre en période de pandémie, même si l'école est fermée. Ce travail en réseaux, qu'il se fasse à l'école ou à distance, nest pas sans rappeler les pratiques de "modération sociale » (Laveault et Yerly, 2017; WyattSmith, Klenowski et Gunn) où les enseignants sont amenés à se concerter pour développer 
une interprétation commune du curriculum, des objectifs d'apprentissage, des critères et des indicateurs de réussite permettant d'attester de la progression des élèves à des fins d'évaluation certificative. Ce travail d'harmonisation sera de plus en plus nécessaire si l'on souhaite réduire au maximum les facteurs pouvant être sources d'inégalités entre les élèves et de fluctuations dans l'interprétation de leurs résultats.

\section{Conclusion}

La pandémie de COVID-19 aura et va encore nécessiter d'importants efforts de solidarité, d'adaptation et de créativité de la part de tous les acteurs de la vie éducative. Mettre en place d'autres manières d'enseigner, d'apprendre et d'évaluer hors des fonctionnements traditionnels aura et va encore demander du temps, un développement professionnel accru et du travail en collaboration pour les enseignants. Sur le plan de l'évaluation, il faudra travailler à établir un équilibre délicat entre les différents rôles de l'évaluation, celui de la certification et celui de soutien à l'apprentissage. La priorité devra se porter vers l'aide aux élèves à risque d'échec et/ou de décrochage et vers l'atténuation des disparités, tout en développant le potentiel de chacun. Les enseignants devront être soutenus dans ce vaste chantier. Les autorités devront également être garantes de la qualité du système (par ex. : des diplômes décernés durant cette période) afin que la pandémie de COVID-19 laisse le moins de traces possible dans le cursus des élèves. La réussite de cet objectif passera par un partenariat encore plus grand entre parents et École. Alors que le temps et les ressources comptent, il est important que tous (gestionnaires, enseignants, parents et élèves) soient solidaires.

\section{Notes}

1 Par commodité, le masculin est utilisé comme générique.

2 Par exemple, en Suisse durant les années 1990, l'abandon des notes a été effectif dans les cantons de Genève et Vaud. Un retour en arrière a été rapidement opéré suite à des oppositions venant de la société civile, et notamment sous l'impulsion d'associations d'enseignants.

\section{Références}

Anderson, L. W. (2002). Curricular Alignment: A Re-Examination, Theory into Practice, 41(4), 255-260.

Birenbaum, M. (2016). Assessment Culture Versus Testing Culture : The Impact on Assessment for Learning. Dans D. Laveault et L. Allal (dir..). Assessment for Learning : Meeting the Challenge of Implementation. Springer.

Butera, F. (2011). La menace des notes. Dans F. Butera, C. Buchs et C. Darnon (dir), L'évaluation, une menace? (p. 45-53). PUF.

Cartier, S. et Butler, D. L. (2016). Comprendre et évaluer l'apprentissage dans des activités complexes. Dans B. Noël et S. Cartier (dir), De la métacognition à l’apprentissage autorégulé (p. 41-54). De Boeck.

Clark, I. (2012). Formative Assessment: Assessment is for self-regulated learning. Educational Psychology Review, 24, 205249.

Crahay, M. (2012). L'école peut-elle être juste et efficace? De l'égalité des chances à l'égalité des acquis. De Boeck.

Earl, L. M. (2012). Assessment as Learning: Using Classroom Assessment to Maximize Student Learning. Corwin Press.

Hadji, C. (2017). Savoir mettre en œuvre une évaluation constructive. Dans A. Bentolila (dir.), L'essentiel de la pédagogie (p. 229-250). Nathan. 
Hattie, J. (2012). Visible learning for teachers: Maximizing impact on learning. Routledge.

Laveault, D. (2007). De la régulation au réglage : étude des dispositifs d'évaluation favorisant l'autorégulation des apprentissages. In L. Allal et L. Mottiez Lopez (Ed.) Régulation des apprentissages en situation scolaire et en formation (pp. 207-234). Bruxelles : De Boeck

Laveault, D., et Allal, L. (2016) Assessment for Learning: Meeting the Challenge of Implementation. Bâle : Springer.

Laveault, D. et Yerly, G. (2017). Modération statistique et modération sociale des résultats scolaires. Approches opposées ou complémentaires? Mesure et évaluation en éducation, 40(2), 91-123

Merle, P. (2014). Faut-il en finir avec les notes ? La Vie des idées, 2, décembre 2014.

Merle, P. (2015). Lécole française et l'invention de la note : Un éclairage historique sur les polémiques contemporaines. Revue française de pédagogie, 193(4), 77-88.

Mottier Lopez, L. et Allal, L. (2008). Le jugement professionnel en évaluation: un acte cognitif et une pratique sociale située. Revue Suisse des sciences de l'éducation, 30(3), 100-200.

RÉCIT (7 juillet 2020). L'après COVID-19... Redémarrer ou réinventer ? Disponible en ligne : https://ecolebranchee.com/apres-covid-19-redemarrer-ou-reinventer/

Roegiers, X. (2004). L'école et l'évaluation, des situations pour évaluer les compétences des élèves. Bruxelles : De Boeck.

Scallon, G. (2004). L évaluation des apprentissages dans une approche par compétences. Montréal : Renouveau pédagogique.

Viau, R. (2009). La motivation en contexte scolaire. De Boeck université.

Wyatt-Smith, C., Klenowski, V., et Gunn, S. (2010). The centrality of teachers' judgement practice in assessment: a study of standards in moderation. Assessment in Education: Principles, Policy et Practice, 17(1), 59-75.

Yerly, G. (2017). Evaluation des apprentissages en classe et évaluation à large échelle. Quel est l'impact des épreuves externes sur les pratiques évaluatives des enseignants? Mesure et Évaluation en éducation, 40(1), 33-60

Yerly, G. et Issaieva, E. (in press). Évaluer les apprentissages au postsecondaire en temps de crise : défis, opportunités et dangers lors de la pandémie de COVID-19. Revue internationale des technologies en pédagogie universitaire

\section{Pour citer cet article}

Yerly, G. et Labeault, D. (2020). Évaluer les apprentissages en contexte de pandémie : aller au-delà de la notation pour soutenir la réussite de tous les élèves. Formation et profession, 28 (4 hors-série), 1-12.

http://dx.doi.org/10.18162/fp.2020.676 